\title{
A!
}

This is an electronic reprint of the original article.

This reprint may differ from the original in pagination and typographic detail.

"Ra'di", Younes; Asadchy, Viktar; Tretyakov, Sergei

\section{One-way transparent sheets}

Published in:

Physical Review B

DOI:

10.1103/PhysRevB.89.075109

Published: 01/01/2014

Document Version

Publisher's PDF, also known as Version of record

Please cite the original version:

"Ra'di", Y., Asadchy, V., \& Tretyakov, S. (2014). One-way transparent sheets. Physical Review B, 89(7), 1-7. [075109]. https://doi.org/10.1103/PhysRevB.89.075109

This material is protected by copyright and other intellectual property rights, and duplication or sale of all or part of any of the repository collections is not permitted, except that material may be duplicated by you for your research use or educational purposes in electronic or print form. You must obtain permission for any other use. Electronic or print copies may not be offered, whether for sale or otherwise to anyone who is not an authorised user. 


\title{
One-way transparent sheets
}

\author{
Y. Ra'di, V. S. Asadchy, and S. A. Tretyakov \\ Department of Radio Science and Engineering, Aalto University, P.O. Box 13000, FI-00076 Aalto, Finland
}

(Received 17 October 2013; revised manuscript received 10 January 2014; published 11 February 2014)

\begin{abstract}
In this paper we introduce the concept of metasurfaces which are fully transparent when looking from one of the two sides of the sheet and have controllable functionalities for waves hitting the opposite side (one-way transparent sheets). We address the question on what functionalities are allowed, considering limitations due to reciprocity and passivity. In particular, we have found that it is possible to realize one-way transparent sheets which have the properties of a twist polarizer in transmission when illuminated from the other side. Also, active one-way transparent sheets with controllable copolarized reflection and transmission from the opposite side are feasible. We show that particular nonreciprocal magnetoelectric coupling inside the sheet is necessary to realize lossless nonactive transparent sheets. Furthermore, we derive the required polarizabilities of constituent dipole particles such that the layers composed of them form one-way transparent sheets. We conclude with design and simulations of an example of a nonreciprocal one-way transparent sheet functioning as an isolating twist polarizer.
\end{abstract}

DOI: 10.1103/PhysRevB.89.075109

\section{INTRODUCTION}

Many novel and elegant device designs in antenna engineering and optics will become possible if we become able to realize electrically (optically) thin layers which have the application-required reflection and transmission coefficients. This need is addressed by inventing various metasurfaces (see reviews in Refs. [1,2]). Metasurfaces, usually realized as electrically and/or magnetically polarizable composite layers, can shape reflected and transmitted wave fronts for required functionalities. Sheets with angle-stable reflection and transmission [3], absorbing sheets [4-6], high-impedance surfaces, including artificial magnetic conductors [7], and various polarization-transforming devices [8-11] are examples of metasurfaces. Recently, thin functional sheets have attracted considerable attention also in optics, since the possibilities to control optical transmission, reflection, and refraction using nonuniform sheets have been understood [2,12-14]. In the majority of studies, the main focus has been on tailoring the transmitted wave while the reflection is kept as low as possible.

Here we study sheets that are totally transparent from one of the two sides (one-way transparent sheets) for normally incident plane waves. The main goal of this study is to find out what functionalities are possible if one-way transparency is required. Can we make the layer fully reflecting or act as a twist polarizer or a phase shifter for plane waves coming from the opposite, nontransparent side? The second related question regards what kind of physical properties the constituents of these sheets must have in order to ensure the desired functionalities. Finally, we present a practical design example of a one-way transparent sheet. Clearly, one-way transparent sheets can have multiple applications due to their "invisibility" for excitations from one side.

Metasurfaces are microscopically structured layers (usually periodic), where the (average) distance between inclusions is smaller than the wavelength in the surrounding media, ensuring that the surfaces do not generate diffraction lobes. For an observer in the far zone the response is that of effectively homogeneous current sheets. Thus, for layers of electrically negligible thickness (metasurfaces) illuminated by normally incident plane waves, the reflected and transmitted waves
PACS number(s): 81.05.Xj, 85.70.Ge, 68.49.-h

are plane waves created by the surface-averaged electric and magnetic current sheets with the surface current densities $\mathbf{J}_{\mathrm{e}}$ and $\mathbf{J}_{\mathrm{m}}$. In composite sheets, the layer has a complicated microstructure, usually containing some electrically small but resonant inclusions (such as complex-shape patches or split rings or small helices). The surface-averaged current densities can be related to the electric and magnetic dipole moments $\mathbf{p}, \mathbf{m}$ induced in each unit cell as $\mathbf{J}_{\mathrm{e}}=\frac{j \omega \mathbf{p}}{S}, \mathbf{J}_{\mathrm{m}}=\frac{j \omega \mathbf{m}}{S}$. Here $S$ is the unit-cell area, and we use the time-harmonic convention $\exp (j \omega t)$. The higher-order multipoles induced in the inclusions do not contribute to the radiated plane-wave fields of the infinite array and we do not need to consider them explicitly. For realizing a one-way transparent sheet we will need to find such structures, where the induced surface-averaged current densities equal zero for illumination from one of the sheet sides but have nontrivial and controllable values if the incidence direction is reversed. In the next section we introduce the model for polarizations induced in the unit cells of metasurfaces which we will study here.

\section{TRANSPARENT ARRAYS OF BIANISOTROPIC UNIT CELLS}

\section{A. Effective polarizability dyadics of particles in periodic arrays}

In order to reveal the most general possible functionalities of one-way transparent sheets, we assume the most general linear relations between the induced polarizations and the fields, the bianisotropic relations. It is convenient to write these relations as the linear relations between the dipole moments of the unit cells and the incident electromagnetic fields, which is equivalent to relating the induced surface current densities to the incident fields:

$$
\left[\begin{array}{l}
\mathbf{p} \\
\mathbf{m}
\end{array}\right]=\left[\begin{array}{ll}
\overline{\bar{\alpha}}_{\mathrm{ee}} & \overline{\bar{\alpha}}_{\mathrm{em}} \\
\overline{\widehat{\alpha}}_{\mathrm{me}} & \overline{\widehat{\alpha}}_{\mathrm{mm}}
\end{array}\right] \cdot\left[\begin{array}{l}
\mathbf{E}_{\mathrm{inc}} \\
\mathbf{H}_{\mathrm{inc}}
\end{array}\right] .
$$

In this paper we deal with two sets of polarizabilities: individual and effective. Individual polarizabilities, denoted as $\alpha_{i j}$ (without hat), define the response of a single particle in free space to the incident electromagnetic fields. Effective (or 
TABLE I. Types of magnetoelectric coupling effects.

\begin{tabular}{ll}
\hline \hline Omega & Chiral \\
$\overline{\bar{\alpha}}_{\mathrm{em}}=\overline{\bar{\alpha}}_{\mathrm{me}}=j \Omega \overline{\bar{J}}_{\mathrm{t}}$ & $\overline{\bar{\alpha}}_{\mathrm{em}}=-\overline{\bar{\alpha}}_{\mathrm{me}}=j \kappa \overline{\bar{I}}_{\mathrm{t}}$ \\
Moving & Tellegen \\
$\overline{\bar{\alpha}}_{\mathrm{em}}=-\overline{\bar{\alpha}}_{\mathrm{me}}=V \overline{\bar{J}}_{\mathrm{t}}$ & $\overline{\bar{\alpha}}_{\mathrm{em}}=\overline{\bar{\alpha}}_{\mathrm{me}}=\chi \overline{\bar{I}}_{\mathrm{t}}$ \\
\hline \hline
\end{tabular}

collective) polarizabilities, denoted as $\widehat{\alpha}_{i j}$ (marked by hat), are the polarizabilities of a particle which is located in an infinite array of particles. Even though the effective polarizabilities show the response of the particle to the incident wave, because the particles are located in an array these polarizabilities also include the effects of lattice interactions. Dual bars denote tensorial (dyadic) quantities. Here we study isotropic (in the plane) thin sheets. The uniaxial symmetry ensures the isotropic response of the metasurfaces for normally incident plane waves of arbitrary polarizations. The orientation of the layer in space is defined by the unit vector $\mathbf{z}_{0}$ orthogonal to its plane. The uniaxial symmetry allows only isotropic response and rotation around the axis $\mathbf{z}_{0}$. Thus, we can write all the polarizabilities in Eq. (1) in the forms

$$
\begin{aligned}
& \overline{\widehat{\alpha}}_{\mathrm{ee}}=\widehat{\alpha}_{\mathrm{ee}}^{\mathrm{co}} \overline{\bar{I}}_{\mathrm{t}}+\widehat{\alpha}_{\mathrm{ee}}^{\mathrm{cr}} \overline{\bar{J}}_{\mathrm{t}}, \quad \overline{\widehat{\alpha}}_{\mathrm{mm}}=\widehat{\alpha}_{\mathrm{mm}}^{\mathrm{co}} \overline{\bar{I}}_{\mathrm{t}}+\widehat{\alpha}_{\mathrm{mm}}^{\mathrm{cr}} \overline{\bar{J}}_{\mathrm{t}}, \\
& \overline{\widehat{\alpha}}_{\mathrm{em}}=\widehat{\alpha}_{\mathrm{em}}^{\mathrm{co}} \overline{\bar{I}}_{\mathrm{t}}+\widehat{\alpha}_{\mathrm{em}}^{\mathrm{cr}} \overline{\bar{J}}_{\mathrm{t}}, \quad \overline{\bar{\alpha}}_{\mathrm{me}}=\widehat{\alpha}_{\mathrm{me}}^{\mathrm{co}} \overline{\bar{I}}_{\mathrm{t}}+\widehat{\alpha}_{\mathrm{me}}^{\mathrm{cr}} \overline{\bar{J}}_{\mathrm{t}},
\end{aligned}
$$

where indices co and cr refer to the symmetric and antisymmetric parts of the corresponding dyadics, respectively. Here $\overline{\bar{I}}_{\mathrm{t}}=$ $\overline{\bar{I}}-\mathbf{z}_{0} \mathbf{z}_{0}$ is the two-dimensional unit dyadic, and $\overline{\bar{J}}_{\mathrm{t}}=\mathbf{z}_{0} \times \overline{\bar{I}}_{\mathrm{t}}$ is the vector-product operator. In the last set of relations it is convenient to separate the coupling coefficients responsible for reciprocal and nonreciprocal coupling processes [15]:

$$
\begin{aligned}
& \overline{\widehat{\alpha}}_{\mathrm{em}}=(\widehat{\chi}-j \widehat{\kappa}) \overline{\bar{I}}_{\mathrm{t}}+(\widehat{V}+j \widehat{\Omega}) \overline{\bar{J}}_{\mathrm{t}}, \\
& \overline{\widehat{\alpha}}_{\mathrm{me}}=(\widehat{\chi}+j \widehat{\kappa}) \overline{\bar{I}}_{\mathrm{t}}+(-\widehat{V}+j \widehat{\Omega}) \overline{\bar{J}}_{\mathrm{t}} .
\end{aligned}
$$

There are two reciprocal classes (chiral $\widehat{\kappa}$ and omega $\widehat{\Omega}$ ) and two nonreciprocal classes ("moving" $\widehat{V}$ and Tellegen $\widehat{\chi}$ ). Note also that for reciprocal particles the electric and magnetic polarizabilities are always symmetric dyadics. The four main types of magnetoelectric coupling are summarized in Table I. The imaginary units in these notations are introduced in order to ensure that all the polarizability components are purely real for lossless particles.

\section{B. Reflection and transmission of plane waves from uniaxial bianisotropic arrays}

We consider array properties for normally incident plane waves. In the following theory of one-way transparent layers, we need to distinguish between illuminations of the sheet from two opposite sides. In the rest of the paper, we use double signs for these two cases, where the top and bottom signs correspond to the incident plane wave propagating in the $-\mathbf{z}_{0}$ and $\mathbf{z}_{0}$ directions, respectively. In the incident plane wave, the electric and magnetic fields satisfy

$$
\mathbf{H}_{\mathrm{inc}}=\mp \frac{1}{\eta_{0}} \mathbf{z}_{0} \times \mathbf{E}_{\mathrm{inc}}=\mp \frac{1}{\eta_{0}} \overline{\bar{J}}_{\mathrm{t}} \cdot \mathbf{E}_{\mathrm{inc}},
$$

where $\eta_{0}=\sqrt{\mu_{0} / \epsilon_{0}}$ is the wave impedance in the isotropic background medium (possibly free space). In terms of the effective polarizabilities, the dipole moments in Eq. (1) can be written as

$$
\left[\begin{array}{l}
\mathbf{p} \\
\mathbf{m}
\end{array}\right]=\left[\begin{array}{c}
\overline{\widehat{\widehat{\alpha}}}_{\mathrm{ee}} \mp \frac{1}{\eta_{0}} \overline{\bar{\alpha}}_{\mathrm{em}} \cdot \overline{\bar{J}}_{\mathrm{t}} \\
\overline{\widehat{\widehat{\alpha}}}_{\mathrm{me}} \mp \frac{1}{\eta_{0}} \overline{\widehat{\widehat{\alpha}}}_{\mathrm{mm}} \cdot \overline{\bar{J}}_{\mathrm{t}}
\end{array}\right] \cdot \mathbf{E}_{\mathrm{inc}} \cdot
$$

Knowing the dipole moments induced in each unit cell and substituting the polarizabilities from (2), we can now write the amplitudes of the reflected and transmitted plane waves as [11]

$$
\begin{aligned}
\mathbf{E}_{\mathrm{r}}= & -\frac{j \omega}{2 S}\left[\eta_{0} \mathbf{p} \mp \mathbf{z}_{0} \times \mathbf{m}\right] \\
= & -\frac{j \omega}{2 S}\left\{\left[\eta_{0} \widehat{\alpha}_{\mathrm{ee}}^{\mathrm{co}} \pm 2 j \widehat{\Omega}-\frac{1}{\eta_{0}} \widehat{\alpha}_{\mathrm{mm}}^{\mathrm{co}}\right] \overline{\bar{I}}_{\mathrm{t}}\right. \\
& \left.+\left[\eta_{0} \widehat{\alpha}_{\mathrm{ee}}^{\mathrm{cr}} \mp 2 \widehat{\chi}-\frac{1}{\eta_{0}} \widehat{\alpha}_{\mathrm{mm}}^{\mathrm{cr}}\right] \overline{\bar{J}}_{\mathrm{t}}\right\} \cdot \mathbf{E}_{\mathrm{inc}}, \\
\mathbf{E}_{\mathrm{t}}= & \mathbf{E}_{\mathrm{inc}}-\frac{j \omega}{2 S}\left[\eta_{0} \mathbf{p} \pm \mathbf{z}_{0} \times \mathbf{m}\right] \\
= & \left\{\left[1-\frac{j \omega}{2 S}\left(\eta_{0} \widehat{\alpha}_{\mathrm{ee}}^{\mathrm{co}} \pm 2 \widehat{V}+\frac{1}{\eta_{0}} \widehat{\alpha}_{\mathrm{mm}}^{\mathrm{co}}\right)\right] \overline{\bar{I}}_{\mathrm{t}}\right. \\
& \left.-\frac{j \omega}{2 S}\left[\eta_{0} \widehat{\alpha}_{\mathrm{ee}}^{\mathrm{cr}} \mp 2 j \widehat{\kappa}+\frac{1}{\eta_{0}} \widehat{\alpha}_{\mathrm{mm}}^{\mathrm{cr}}\right] \overline{\bar{J}}\right) \cdot \mathbf{E}_{\mathrm{inc}},
\end{aligned}
$$

in which $S$ is the unit-cell area. Using these relations, we next study transparent layers.

\section{General conditions for totally transparent layers}

By definition, a transparent layer must not change the amplitude and phase of incident waves, that is,

$$
\mathbf{E}_{\mathrm{r}}=0, \quad \mathbf{E}_{\mathrm{t}}=\mathbf{E}_{\mathrm{inc}} .
$$

From (6) and (7) we find the necessary conditions for a transparent array of particles in the form

$$
\begin{gathered}
\eta_{0} \widehat{\alpha}_{\mathrm{ee}}^{\mathrm{co}} \pm 2 j \widehat{\Omega}-\frac{1}{\eta_{0}} \widehat{\alpha}_{\mathrm{mm}}^{\mathrm{co}}=0, \\
\eta_{0} \widehat{\alpha}_{\mathrm{ee}}^{\mathrm{cr}} \mp 2 \widehat{\chi}-\frac{1}{\eta_{0}} \widehat{\alpha}_{\mathrm{mm}}^{\mathrm{cr}}=0, \\
\eta_{0} \widehat{\alpha}_{\mathrm{ee}}^{\mathrm{co}} \pm 2 \widehat{V}+\frac{1}{\eta_{0}} \widehat{\alpha}_{\mathrm{mm}}^{\mathrm{co}}=0, \\
\eta_{0} \widehat{\alpha}_{\mathrm{ee}}^{\mathrm{cr}} \mp 2 j \widehat{\kappa}+\frac{1}{\eta_{0}} \widehat{\alpha}_{\mathrm{mm}}^{\mathrm{cr}}=0 .
\end{gathered}
$$

As above, the double signs correspond to the two opposite incidence directions. Clearly, these conditions ensure that the surface-averaged induced electric and magnetic current densities equal zero [see Eqs. (6) and (7)].

First, it is obvious that the conditions for two-way transparency allow only trivial solution: In this case all the polarizability components must equal zero. Indeed, if we demand that conditions (9) are satisfied for both choices of the \pm sign, so that the sheet looks transparent from both sides, then all the magnetoelectric coefficients must be zero. Next, we see immediately that in that case all the other polarizabilities must 
also vanish. We stress that this does not imply that the sheet is simply absent: Zero dipole moments in each unit cell mean only that the surface-averaged electric and magnetic current densities are zero. For example, a low-loss frequency-selective surface is transparent from both sides at the parallel resonance of the unit cell, although strong currents are induced in the structure. It is quite a simple but interesting result. In particular, it implies that sheets which are fully transparent only from one side must exhibit electromagnetic coupling inside inclusions, or the sheet will be transparent from both sides. Note that this general conclusion holds also for nonreciprocal sheets.

What will be the properties of an array which is transparent from one side for waves coming from the other side? We expect that using different particles (reciprocal and nonreciprocal) we should be able to control the response seen from the other side of the sheet. Suppose that a grid of particles is set to be transparent for $+\mathbf{z}_{0}$-directed incident waves. Then, using (9), we can express the electric and magnetic polarizabilities in terms of the magnetoelectric parameters:

$$
\begin{aligned}
& \eta_{0} \widehat{\alpha}_{\mathrm{ee}}^{\mathrm{co}}-\frac{1}{\eta_{0}} \widehat{\alpha}_{\mathrm{mm}}^{\mathrm{co}}=2 j \widehat{\Omega}, \\
& \eta_{0} \widehat{\alpha}_{\mathrm{ee}}^{\mathrm{cr}}-\frac{1}{\eta_{0}} \widehat{\alpha}_{\mathrm{mm}}^{\mathrm{cr}}=-2 \widehat{\chi}, \\
& \eta_{0} \widehat{\alpha}_{\mathrm{ee}}^{\mathrm{co}}+\frac{1}{\eta_{0}} \widehat{\alpha}_{\mathrm{mm}}^{\mathrm{co}}=2 \widehat{V}, \\
& \eta_{0} \widehat{\alpha}_{\mathrm{ee}}^{\mathrm{cr}}+\frac{1}{\eta_{0}} \widehat{\alpha}_{\mathrm{mm}}^{\mathrm{cr}}=-2 j \widehat{\kappa} .
\end{aligned}
$$

From (6) and (7), the reflected and transmitted fields for the wave coming from the nontransparent side $\left(-\mathbf{z}_{0}\right.$-directed wave) can be expressed in terms of the magnetoelectric parameters only:

$$
\begin{gathered}
\mathbf{E}_{\mathrm{r}}=\frac{j 2 \omega}{S}\left(-j \widehat{\Omega} \overline{\bar{I}}_{\mathrm{t}}+\widehat{\chi} \overline{\bar{J}}_{\mathrm{t}}\right) \cdot \mathbf{E}_{\mathrm{inc}} \\
\mathbf{E}_{\mathrm{t}}=\left[\left(1-\frac{j 2 \omega}{S} \widehat{V}\right) \overline{\bar{I}}_{\mathrm{t}}-\frac{2 \omega}{S} \widehat{\kappa} \overline{\bar{J}}_{\mathrm{t}}\right] \cdot \mathbf{E}_{\mathrm{inc}} .
\end{gathered}
$$

Now, we can study possible responses of reciprocal and nonreciprocal one-way transparent sheets.

\section{Reciprocal one-way transparent sheets}

Let us first consider arrays of reciprocal unit cells (omega and chiral bianisotropic coupling). For reciprocal particles, the electric and magnetic polarizabilities are symmetric dyadics $\left(\widehat{\alpha}_{\mathrm{ee}}^{\mathrm{cr}}=0, \widehat{\alpha}_{\mathrm{mm}}^{\mathrm{cr}}=0\right)$, and the parameters of the nonreciprocal magnetoelectric coupling vanish $(\widehat{\chi}=\widehat{V}=0)$. The last relation in Eq. (10) tells that the chirality parameter $\widehat{\kappa}$ is also zero. This ensures that the transmission coefficient from the other side (12) equals unity, as it should be due to reciprocity.

From the other relations (10) we find that

$$
\eta_{0} \widehat{\alpha}_{\mathrm{ee}}^{\mathrm{co}}=j \widehat{\Omega}=-\frac{1}{\eta_{0}} \widehat{\alpha}_{\mathrm{mm}}^{\mathrm{co}}
$$

and, using (11), the reflected field for the waves coming from the other side can be written as

$$
\mathbf{E}_{\mathrm{r}}=\frac{2 \omega}{S} \widehat{\Omega} \mathbf{E}_{\text {inc }} .
$$

If the array is passive, then the absolute value of this reflection coefficient must equal zero, because the transmission coefficient is unity from both sides (due to reciprocity). Thus, the omega coupling coefficient in passive reciprocal one-way transparent sheets must be zero for all nonzero frequencies, and we end up with the trivial solution when all the polarizabilities are zero. However, if the inclusions can be active, we see that reciprocal layers can be transparent from one side, while the copolarized reflection from the other side can be controlled by the value of the omega coupling. Note that this is the only possible functionality even for active inclusions: The requirement of reciprocity is very limiting, because it sets the transmission coefficient to be unity from both sides. In particular, this does not allow chirality in the particles, and, thus, no polarization transformation is possible in isotropic reciprocal one-way transparent sheets.

\section{E. Nonreciprocal one-way transparent sheets}

From Eqs. (11) and (12) it appears that the use of nonreciprocal particles, in principle, allows full control over co- and cross-polarized reflection and transmission coefficients of one-way transparent sheets (passivity limitations are discussed below). To find out what polarizabilities are required for any desired functionality, one can start from the required reflection and transmission coefficients and find the corresponding magnetoelectric parameters. For example, if we would like to realize a one-way transparent twist polarizer in transmission $\left(\mathbf{E}_{\mathrm{t}}=\mathbf{z}_{0} \times \mathbf{E}_{\mathrm{inc}}=\overline{\bar{J}}_{\mathrm{t}} \cdot \mathbf{E}_{\mathrm{inc}}\right)$, the required values of the coupling parameters read

$$
\widehat{\kappa}=-\frac{S}{2 \omega}, \quad \widehat{V}=-j \frac{S}{2 \omega}, \quad \widehat{\Omega}=\widehat{\chi}=0 .
$$

The corresponding electric and magnetic polarizabilities follow from (10):

$$
\begin{aligned}
& \eta_{0} \widehat{\alpha}_{\mathrm{ee}}^{\mathrm{co}}=\frac{1}{\eta_{0}} \widehat{\alpha}_{\mathrm{mm}}^{\mathrm{co}}=\widehat{V}=-j \frac{S}{2 \omega}, \\
& \eta_{0} \widehat{\alpha}_{\mathrm{ee}}^{\mathrm{cr}}=\frac{1}{\eta_{0}} \widehat{\alpha}_{\mathrm{mm}}^{\mathrm{cr}}=-j \widehat{\kappa}=j \frac{S}{2 \omega} .
\end{aligned}
$$

Note that the magnitudes of all the required normalized polarizabilities are equal, and this provides one of the examples of extreme response of balanced bianisotropic particles [16]. It is easy to check from (5) that in this case of zero reflection the induced electric and magnetic dipoles of each unit cell form Huygens pairs, radiating only in the forward direction, as it should be for any nonreflecting sheet (see examples in Refs. $[11,13,14])$.

Let us next discuss the limitations which follow from the energy conservation. Considering ideally lossless structures, it is easy to see that any lossless one-way transparent sheet must have zero reflections from both sides. This is because the total power of two waves (the reflected wave and the wave transmitted from the transparent side) is not equal to the sum of the powers of these two waves due to their interference. 
Thus, lossless one-way transparent sheets allow control only over the transmission coefficients for waves coming from the opposite direction (the polarization state and phase delays of transmitted waves can be engineered with complete freedom). If we want to control the back reflection in one-way transparent sheets, the structure must be lossy or active. First, let us suppose that the layer is passive and lossy. In this case from the nontransparent side we can have partial reflection and partial transmission, but still it is not possible to have full control over the reflection and transmission properties. Because of the interference between the two waves propagating away from the structure at its nontransparent side, there is a limit for the reflected power dictated by the energy conservation. Full control over reflection can be achieved only with active structures. Conceptually, it is possible to fully absorb the power incident from the nontransparent side and produce secondary ("reflected") waves using active elements. As another example of passivity limitations, let us assume that only the Tellegen parameter $\widehat{\chi}$ is nonzero while all the other coupling coefficients are zero. In this case we see that the transmission coefficient from both sides equals unity and conclude that in this case it is possible to control the cross-polarized reflection only if the particles are active.

An interesting case is the case of a "moving" grid $(\widehat{V} \neq 0)$. We can set $\widehat{\Omega}=\widehat{\chi}=0$, so that the reflection coefficient is zero and the induced current sheets form a Huygens' pair. Upon substitution of $\widehat{\Omega}=\widehat{\chi}=0$ in Eq. (10), we see that the electric and magnetic polarizabilities are balanced:

$$
\eta_{0} \widehat{\alpha}_{\mathrm{ee}}^{\mathrm{co}}=\frac{1}{\eta_{0}} \widehat{\alpha}_{\mathrm{mm}}^{\mathrm{co}}, \quad \eta_{0} \widehat{\alpha}_{\mathrm{ee}}^{\mathrm{cr}}=\frac{1}{\eta_{0}} \widehat{\alpha}_{\mathrm{mm}}^{\mathrm{cr}} .
$$

We conclude that we can fully control the transmission coefficient choosing the values of $\widehat{V}$ and $\widehat{\kappa}$ [each of these two parameters will uniquely define the values in Eq. (17)], maintaining the property of zero reflection (Huygens' layer). The only limitation on the transmission coefficient values comes from passivity: The total amplitude of the transmitted field should not be larger than the amplitude of the incident field. One of the interesting limiting cases is the case of nonchiral moving arrays. Setting $\widehat{\kappa}=0$, we find the required effective polarizabilities as

$$
\eta_{0} \widehat{\alpha}_{\mathrm{ee}}^{\mathrm{co}}=\widehat{V}=\frac{1}{\eta_{0}} \widehat{\alpha}_{\mathrm{mm}}^{\mathrm{co}}, \quad \eta_{0} \widehat{\alpha}_{\mathrm{ee}}^{\mathrm{cr}}=\frac{1}{\eta_{0}} \widehat{\alpha}_{\mathrm{mm}}^{\mathrm{cr}}=0,
$$

and, using (11) and (12), the reflection and transmission coefficients for the wave coming from the other side can be written as

$$
\mathbf{E}_{\mathrm{r}}=0, \quad \mathbf{E}_{\mathrm{t}}=\left(1-\frac{j 2 \omega}{S} \widehat{V}\right) \mathbf{E}_{\mathrm{inc}} .
$$

One can see that the sheet of moving particles can be designed to work as a completely transparent layer from one side and a partially transparent layer from the other side (with controllable amplitude and phase of the transmitted field). In the special case of a balanced and lossy layer the sheet is transparent from one side and acts as a perfect absorber from the other side [6].

\section{REQUIREMENTS FOR INDIVIDUAL POLARIZABILITIES OF UNIT CELLS}

The above theory gives the required conditions for effective (collective) polarizabilities of unit cells forming one-way transparent sheets. These parameters connect the induced electric and magnetic dipole moments to the incident electric and magnetic fields; see (1). Thus, the effective polarizabilities depend not only on the individual particles but also on electromagnetic coupling between particles in the infinite array. Here we use the known theory of reflection and transmission in infinite dipole arrays (e.g., [17]) to find the corresponding requirements on the polarizabilities of individual particles in free space. This is necessary to approach the problem of the particle design (finding the inclusion shape and sizes which provide the desired response of the whole array). To characterize individual particles, we consider their response to the local electromagnetic fields, which exist at the position of one reference particle:

$$
\left[\begin{array}{l}
\mathbf{p} \\
\mathbf{m}
\end{array}\right]=\left[\begin{array}{cc}
\overline{\bar{\alpha}}_{\mathrm{ee}} & \overline{\bar{\alpha}}_{\mathrm{em}} \\
\overline{\bar{\alpha}}_{\mathrm{me}} & \overline{\bar{\alpha}}_{\mathrm{mm}}
\end{array}\right] \cdot\left[\begin{array}{l}
\mathbf{E}_{\mathrm{loc}} \\
\mathbf{H}_{\mathrm{loc}}
\end{array}\right] .
$$

Since the grid is excited by plane-wave fields which are uniform in the array plane (normal incidence), the induced dipole moments are the same for all particles. The local fields exciting the particles are the sums of the external incident fields and the interaction fields caused by the induced dipole moments in all other particles,

$$
\mathbf{E}_{\mathrm{loc}}=\mathbf{E}_{\mathrm{inc}}+\beta_{\mathrm{e}} \mathbf{p}, \quad \mathbf{H}_{\mathrm{loc}}=\mathbf{H}_{\mathrm{inc}}+\beta_{\mathrm{m}} \mathbf{m},
$$

where $\beta_{\mathrm{e}}$ and $\beta_{\mathrm{m}}$ are the interaction constants that describe the effect of the entire array on a single inclusion. These dyadic coefficients are proportional to the two-dimensional unit dyadic $\overline{\bar{I}}_{\mathrm{t}}$. Explicit analytical expression for the interaction constants can be found in Ref. [15].

Because all of the dipoles are in the same plane, the induced magnetic dipoles do not produce any electric interaction field in the tangential plane, and vice versa (see [18]). Expressing the incident fields in Eq. (1) in terms of the local fields and the interaction constants (21) we can find the polarizabilities of the individual unit cells in terms of the required collective polarizabilities. In the general case of uniaxial polarizabilities these expressions can be found in Ref. [11]. As an example, let us study the case of a one-way transparent nonchiral "moving" sheet [the required effective polarizabilities are given by (18)]. In this case the relations between the individual and collective polarizabilities can be written as

$$
\begin{gathered}
\overline{\bar{\alpha}}_{\mathrm{ee}}=\frac{\alpha_{\mathrm{ee}}^{\mathrm{co}}-\beta_{\mathrm{m}}\left(\alpha_{\mathrm{ee}}^{\mathrm{co}} \alpha_{\mathrm{mm}}^{\mathrm{co}}-V^{2}\right)}{1-\left(\alpha_{\mathrm{ee}}^{\mathrm{co}} \beta_{\mathrm{e}}+\alpha_{\mathrm{mm}}^{\mathrm{co}} \beta_{\mathrm{m}}\right)+\beta_{\mathrm{e}} \beta_{\mathrm{m}}\left(\alpha_{\mathrm{ee}}^{\mathrm{co}} \alpha_{\mathrm{mm}}^{\mathrm{co}}-V^{2}\right)} \overline{\bar{I}}_{\mathrm{t}}, \\
\overline{\widehat{\alpha}}_{\mathrm{mm}}=\frac{\alpha_{\mathrm{mm}}^{\mathrm{co}}-\beta_{\mathrm{e}}\left(\alpha_{\mathrm{ee}}^{\mathrm{co}} \alpha_{\mathrm{mm}}^{\mathrm{co}}-V^{2}\right)}{1-\left(\alpha_{\mathrm{ee}}^{\mathrm{co}} \beta_{\mathrm{e}}+\alpha_{\mathrm{mm}}^{\mathrm{co}} \beta_{\mathrm{m}}\right)+\beta_{\mathrm{e}} \beta_{\mathrm{m}}\left(\alpha_{\mathrm{ee}}^{\mathrm{co}} \alpha_{\mathrm{mm}}^{\mathrm{co}}-V^{2}\right)} \overline{\bar{I}}_{\mathrm{t}}, \\
\widehat{V} \overline{\bar{J}}_{\mathrm{t}}=\frac{V}{1-\left(\alpha_{\mathrm{ee}}^{\mathrm{co}} \beta_{\mathrm{e}}+\alpha_{\mathrm{mm}}^{\mathrm{co}} \beta_{\mathrm{m}}\right)+\beta_{\mathrm{e}} \beta_{\mathrm{m}}\left(\alpha_{\mathrm{ee}}^{\mathrm{co}} \alpha_{\mathrm{mm}}^{\mathrm{co}}-V^{2}\right)} \overline{\bar{J}}_{\mathrm{t}} .
\end{gathered}
$$


The necessary conditions for the polarizabilities of the single moving particle can be found substituting (22) in conditions (18) as

$$
\eta_{0} \alpha_{\mathrm{ee}}^{\mathrm{co}}=V=\frac{1}{\eta_{0}} \alpha_{\mathrm{mm}}^{\mathrm{co}}, \quad \alpha_{\mathrm{ee}}^{\mathrm{cr}}=\alpha_{\mathrm{mm}}^{\mathrm{cr}}=0 .
$$

These conditions are the balance conditions for individual particles. Interestingly, conditions (23) were obtained in Ref. [19] as the conditions for zero total scattering from small single uniaxial nonreciprocal particles.

Finally, we note that the required reciprocal magnetoelectric coupling in particles can be realized using proper shaping of metal or dielectric inclusions (helical shapes for chiral particles and, for example, the shape of the letter $\Omega$ for omega coupling). Nonreciprocal coupling requires the use of nonreciprocal components, such as magnetized ferrite, plasma, or active components, e.g., amplifiers. For details, we refer to $[15,20]$ and references therein.

\section{EXAMPLE OF A ONE-WAY TRANSPARENT SHEET COMPOSED OF NONRECIPROCAL BIANISOTROPIC PARTICLES}

In this section, we present a realizable design of a one-way transparent sheet acting as a twist polarizer in transmission for the wave incident from the nontransparent side. As a nonreciprocal particle, we use the particle possessing "chiral-moving" coupling (bianisotropy parameters $\widehat{\Omega}=\widehat{\chi}=0$ ) presented in Ref. [21]. As it is shown in Fig. 1, the particle includes a ferrite sphere magnetized by external bias field and coupled to metal elements. Recently, polarizabilities of this particle were extracted analytically and numerically [22]. Considering the polarizabilities of this particle at the resonance frequency, we note that its electric and magnetic polarizabilities become purely imaginary while the chirality parameter is real (as expected, because all the reactances are compensated and only dissipative terms remain for the particle at resonance). This apparently contradicts to the requirements of balanced effective polarizabilities (16) for a one-way transparent twist polarizer. However, we note that the resonant-particle polarizability can

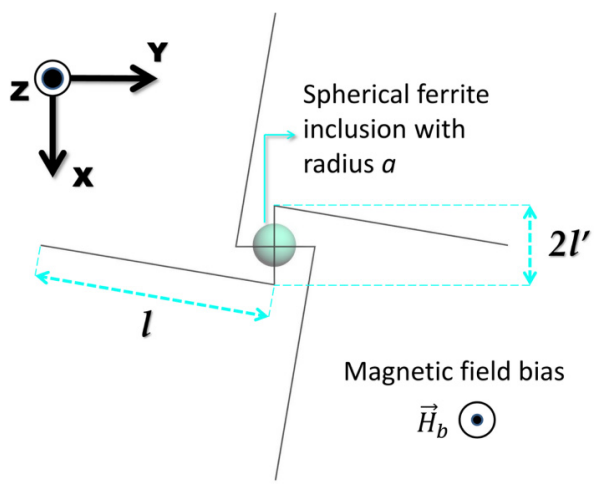

FIG. 1. (Color online) Geometry of a "chiral-moving" particle. The external magnetic field bias is along the $\mathbf{z}_{0}$ axis. satisfy the following similar conditions:

$$
\begin{gathered}
\eta_{0} \widehat{\alpha}_{\mathrm{ee}}^{\mathrm{co}}=\frac{1}{\eta_{0}} \widehat{\alpha}_{\mathrm{mm}}^{\mathrm{co}}=\widehat{V}=-j \frac{S}{2 \omega}, \\
\eta_{0} \widehat{\alpha}_{\mathrm{ee}}^{\mathrm{cr}}=\frac{1}{\eta_{0}} \widehat{\alpha}_{\mathrm{mm}}^{\mathrm{cr}}=-j \widehat{\kappa}=-\frac{S}{2 \omega} .
\end{gathered}
$$

Conditions (24) and (25) are related to the case of a one-way transparent sheet which acts as a twist polarizer with additional $90^{\circ}$ phase shift for the wave incident from the nontransparent side $\left(\mathbf{E}_{\mathrm{t}}=j \overline{\bar{J}}_{\mathrm{t}} \cdot \mathbf{E}_{\mathrm{inc}}\right)$, and we proceed with designing such device.

We use the numerical method [23] (see also Ref. [22]) which allows us to extract polarizabilities of an arbitrary polarizable particle. Deriving the individual polarizabilities of a single particle utilizing this method and using (22) (which relates the effective polarizabilities to the individual ones), we can optimize the dimensions of the particle to realize the required effective polarizabilities (16). The optimized dimensions of the particle (the target frequency is about $2 \mathrm{GHz}$ ) read $l=18 \mathrm{~mm}, l^{\prime}=3 \mathrm{~mm}, a=1.65 \mathrm{~mm}\left(S=a^{2}\right.$ is cell area), and the radius of the wire is $r_{0}=0.05 \mathrm{~mm}$. Material of the metal elements is copper and the ferrite material is yttrium iron garnet. The properties of the ferrite material are the relative permittivity $\epsilon_{r}=15$, the dielectric loss tangent $\tan \delta=10^{-4}$, saturation magnetization $M_{S}=1780 \mathrm{G}$, and the full resonance linewidth $\Delta H=0.2 \mathrm{Oe}$ (measured at $9.4 \mathrm{GHz}$ ). The internal bias field is $H_{b}=9626 \mathrm{~A} / \mathrm{m}$, corresponding to the desired resonance frequency. Simulated individual and effective polarizabilities (for a grid with $S=1482.25 \mathrm{~mm}^{2}$ ) of the optimized particle are shown in Figs. 2 and 3, respectively. These figures exhibit fairly balanced electric and magnetic response in terms of individual and effective polarizabilities.

We verify the operation of the sheet by full-wave simulations using the Ansoft High Frequency Structure
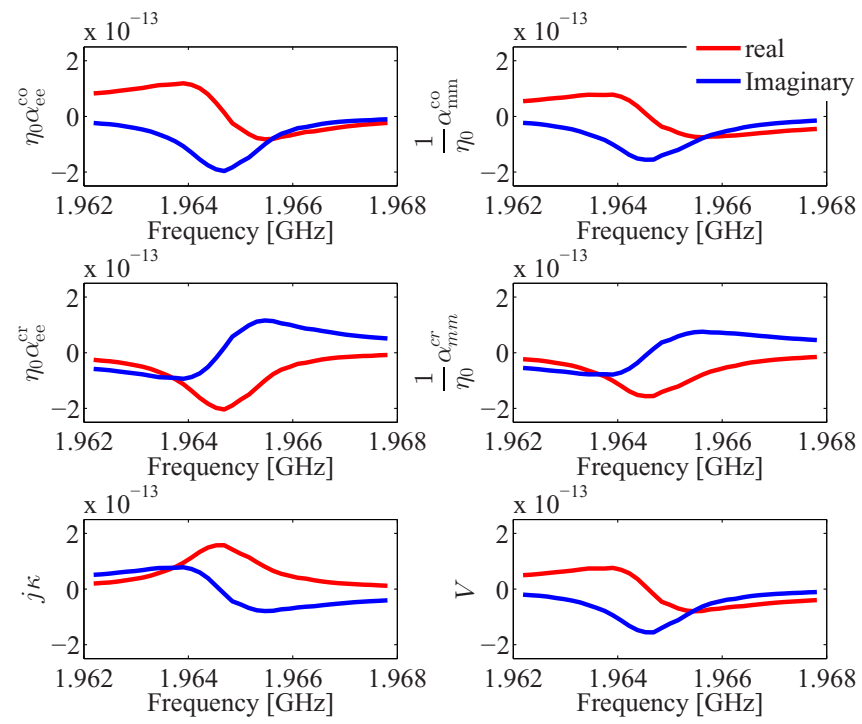

FIG. 2. (Color online) Simulated polarizabilities of an individual particle. 

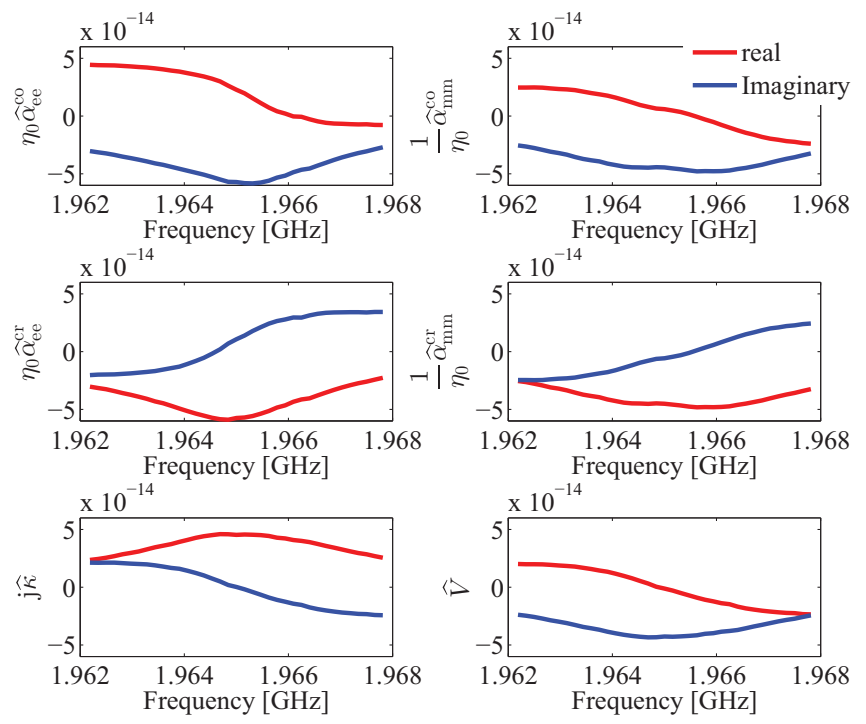

FIG. 3. (Color online) Simulated effective polarizabilities of the grid.

Simulator. Periodic boundary conditions are used to calculate the reflection and transmission coefficients of the sheet made of these particles. Simulated co- and cross-polarized reflection and transmission coefficients for the wave incident from the transparent and nontransparent sides are shown in Fig. 4.
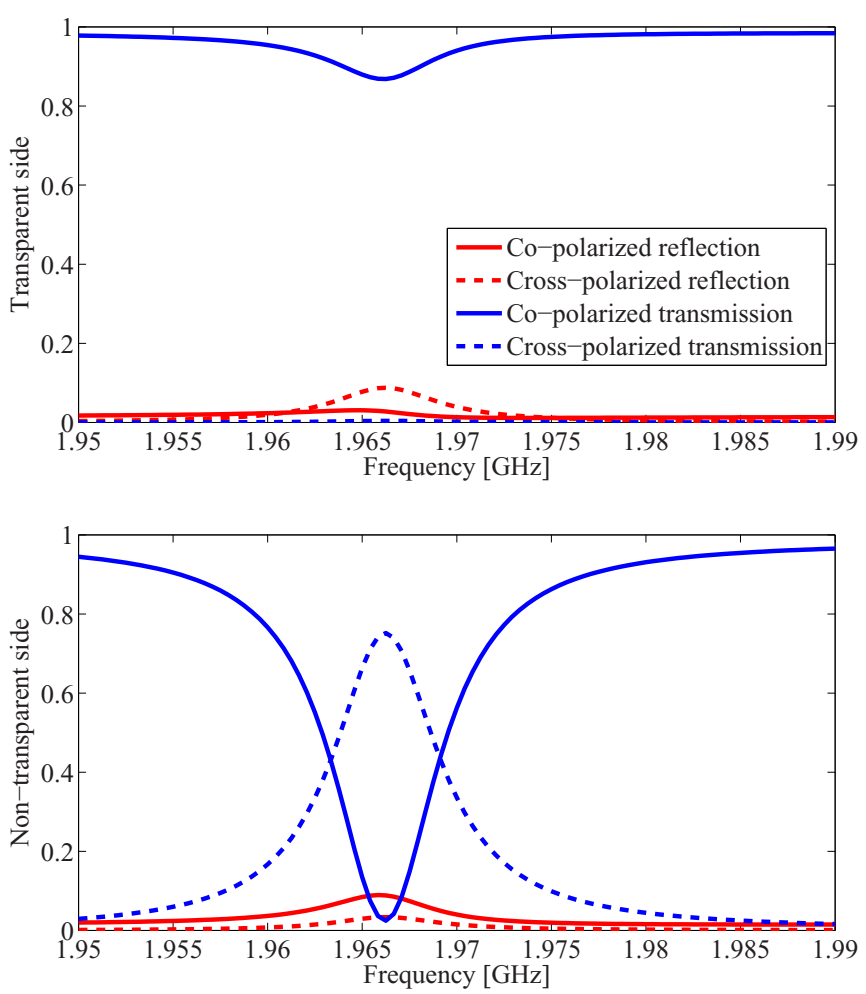

FIG. 4. (Color online) Simulated reflection and transmission (in terms of intensity) for the sheet when the incident wave propagates along the (a) $+\mathbf{z}_{0}$ axis and (b) $-\mathbf{z}_{0}$ axis.
As is seen from Fig. 4, the designed composite sheet transmits $87 \%$ of the incident wave power, propagating along the transparent direction. At the same time, it transmits $75 \%$ of the wave power (in cross polarization) when the layer is illuminated from the nontransparent side. Thus, the sheet acts as a one-way transparent layer and as a twist polarizer with a $90^{\circ}$ phase shift from the nontransparent side, as was theoretically predicted. Nonideal magnitude of the cross-polarized transmitted wave can be explained by inevitable absorption loss inside the ferrite sphere and copper wires (about 13\%). Also, some small reflection exists due to parasitic weak omega and Tellegen coupling effects in the particle (about 12\%).

The designed sheet is a nonreciprocal analogy of the device proposed in Ref. [11], which consists of reciprocal chiral particles and acts as a twist polarizer for both directions of incidence. Nonreciprocal electromagnetic coupling allows us to obtain dramatically different response for the opposite incident directions. The proposed composite sheet based on chiral-moving particles exhibits the target electromagnetic properties of a one-way transparent sheet and has realistic parameters allowing practical realizations.

\section{CONCLUSION}

Although it is not possible to realize a fully transparent sheet except the trivial case of zero averaged induced surface currents, we have shown that it is possible to realize one-way transparent sheets. In these structures, the polarizabilities of unit cells are different from zero, but they are balanced in such a way that the averaged induced currents are zero for illumination from one of the two sides of the sheet. However, the response to plane waves illuminating the opposite side of the sheet is nontrivial and can be controlled by design of the metasurface microstructure. Electromagnetic coupling (bianisotropy) inside unit cells of the metasurface is a necessary condition for one-way transparent layers. If we are limited to the case of lossless sheets with passive particles, then one-way transparency necessarily requires nonreciprocal coupling and is impossible with chiral and omega particles. It was shown that presence of moving coupling is necessary to make a lossless nonactive one-way transparent sheet. In particular, it has been shown that nonreciprocal coupling effects make it possible to realize a one-way transparent sheet which acts as a twist polarizer in transmission when illuminated from the nontransparent side. Another possible device is a one-way transparent phase-shifting sheet. If active particles are allowed, our possibilities to control electromagnetic response from the opposite side of the sheet are extended: There is no restriction on the amplitude of reflection and transmission for the wave coming from the nontransparent side. Also, omega coupling becomes allowed and makes it possible to realize a one-way transparent sheet with controllable copolarized reflection from the opposite side. Required effective and individual polarizabilities of bianisotropic particles as components of a one-way transparent layer have been derived. Finally, we have shown a realistic design of a nonreciprocal one-way transparent sheet and simulated its performance parameters. 
[1] C. L. Holloway, E. F. Kuester, J. A. Gordon, J. O'Hara, J. Booth, and D. R. Smith, IEEE Antennas Propag. Mag. 54, 10 (2012).

[2] A. V. Kildishev, A. Boltasseva, and V. M. Shalaev, Science 339, 1232009 (2013).

[3] J. A. Gordon, C. L. Holloway, and A. Dienstfrey, IEEE Antennas Wireless Propag. Lett. 8, 1127 (2009).

[4] Y. Avitzour, Y. A. Urzhumov, and G. Shvets, Phys. Rev. B 79, 045131 (2009).

[5] C. M. Watts, X. Liu, and W. J. Padilla, Adv. Mater. 24, OP98 (2012).

[6] Y. Ra'di, V. S. Asadchy, and S. A. Tretyakov, IEEE Trans. Antennas Propag. 61, 4606 (2013).

[7] D. Sievenpiper, L. Zhang, R. F. J. Broas, N. G. Alexopoulos, and E. Yablonovich, IEEE Trans. Microwave Theory Tech. 47, 2059 (1999).

[8] I. V. Semchenko, S. A. Khakhomov, and A. L. Samofalov, J. Commun. Technol. Electron. 52, 850 (2007).

[9] M. Euler, V. Fusco, R. Cahill, and R. Dickie, IET Microwaves, Antennas Propag. 4, 1764 (2010).

[10] Y. Zhao and A. Alù, Phys. Rev. B 84, 205428 (2011).

[11] T. Niemi, A. Karilainen, and S. Tretyakov, IEEE Trans. Antennas Propag. 61, 3102 (2013).

[12] N. Yu, P. Genevet, M. A. Kats, F. Aieta, J.-P. Tetienne, F. Capasso, and Z. Gaburro, Science 334, 333 (2011).

[13] C. Pfeiffer and A. Grbic, Phys. Rev. Lett. 110, 197401 (2013).
[14] F. Monticone, N. M. Estakhri, and A. Alù, Phys. Rev. Lett. 110, 203903 (2013).

[15] A. N. Serdyukov, I. V. Semchenko, S. A. Tretyakov, and A. Sihvola, Electromagnetics of Bi-anisotropic Materials: Theory and Applications (Gordon and Breach Science, Amsterdam, 2001).

[16] Y. Ra'di and S. A. Tretyakov, New J. Phys. 15, 053008 (2013).

[17] S. A. Tretyakov, Analytical Modeling in Applied Electromagnetics (Artech House, Norwood, MA, 2003).

[18] V. V. Yatsenko, S. I. Maslovski, S. A. Tretyakov, S. L. Prosvirnin, and S. Zouhdi, IEEE Trans. Antennas Propag. 51, 2 (2003).

[19] J. Vehmas, Y. Ra'di, A. O. Karilainen, and S. A. Tretyakov, IEEE Trans. Antennas Propag. 61, 3747 (2013).

[20] I. Lindell, A. Sihvola, S. Tretyakov, and A. Viitanen, Electromagnetic Waves in Chiral and Bi-isotropic Media (Artech House, Norwood, MA, 1994).

[21] S. A. Tretyakov, Microwave Opt. Technol. Lett. 19, 365 (1998).

[22] M. S. Mirmoosa, Master's thesis, Department of Radio Science and Engineering, Aalto University, 2013, https://aaltodoc.aalto.fi/handle/123456789/11111.

[23] I. A. Faniayeu, V. S. Asadchy, I. V. Semchenko, and S. A. Khakhomov, in Proceedings of Metamaterials '2012: The Sixth International Congress on Advanced Electromagnetic Materials in Microwaves and Optics, St. Petersburg, Russia 17-22 September, 2012 (Metamorphose VI, Espoo, Finland, 2012), pp. 323-326. 